\title{
Prognostic value of CA 125 in ovarian cyst fluid of patients with epithelial ovarian cancer
}

\author{
EVA KOLWIJCK $^{1 *}$, PAUL N. SPAN ${ }^{2 *}$, CHRIS M.G. THOMAS ${ }^{1,2}$, JOHAN BULTEN $^{3}$, \\ FRED C.G.J. SWEEP ${ }^{2}$ and LEON F.A.G. MASSUGER ${ }^{1}$ \\ ${ }^{1}$ Department of Obstetrics and Gynecology, ${ }^{2}$ Department of Chemical Endocrinology, ${ }^{3}$ Department of Pathology \\ Radboud University Nijmegen Medical Centre, P.O. Box 9101, 6500 HB Nijmegen, The Netherlands
}

Received September 27, 2009; Accepted October 23, 2009

DOI: 10.3892/or_00000672

\begin{abstract}
Most ovarian tumors contain ovarian cyst fluid (oCF) which can be easily obtained during surgery. This is the first study that explored if CA 125 in oCF could be of prognostic value for patients with epithelial ovarian cancer (EOC). Of 54 patients with primary EOC, oCF and preoperative serum were collected and clinicopathological data were retrospectively obtained. CA 125 was measured with the commercially available CA 125 assay. CA 125 in oCF ( $\mathrm{n}=54$, median: 55,500 U/ml, range: 590-10,200,000 $\mathrm{U} / \mathrm{ml}$ ) was always higher than in the corresponding serum $(\mathrm{n}=51$, median: $179 \mathrm{U} / \mathrm{ml}$, range: $13-11,000 \mathrm{U} / \mathrm{ml})(\mathrm{p}<0.001)$ and values were moderately correlated $(\mathrm{R}=0.337, \mathrm{p}=0.016)$. CA 125 in oCF was associated with histology $(\mathrm{p}<0.001)$ and tumor grade $(p=0.038)$. High levels of oCF CA 125 (>median) were significantly associated with a poor diseasefree survival (DFS) $(\log$-rank $\mathrm{p}=0.002$ and $\mathrm{p}=0.005$ univariate Cox-regression). Other factors associated with a poor DFS in univariate analysis were advanced FIGO stage, suboptimal debulking (both $\mathrm{p}<0.001$ ), high tumor grade $(\mathrm{p}=0.025)$, serous histology $(\mathrm{p}=0.003)$ and high serum (>media) CA 125 ( $\mathrm{p}=0.009)$. In multivariate analysis, only FIGO stage was of independent predictive value. These findings indicate that, although high levels of oCF CA 125 were significantly associated with a poor survival of EOC patients, CA 125 in oCF was not of independent predictive value and might therefore not be useful as a prognostic biomarker for EOC.
\end{abstract}

Correspondence to: Dr Eva Kolwijck, Department of Obstetrics and Gynaecology, Radboud University Nijmegen Medical Centre, P.O. Box 9101, 6500 HB Nijmegen, The Netherlands

E-mail: e.kolwijck@obgyn.umcn.nl

*Contributed equally

Key words: CA 125, biomarker, ovarian cancer, ovarian carcinoma, cyst fluid

\section{Introduction}

Epithelial ovarian cancer (EOC) is the leading cause of death from gynecological malignancies in developed countries. Due to the absence of specific signs and symptoms in an early stage of disease, the majority of patients are diagnosed when the tumor has already metastasized to the upper abdomen with a subsequent 5-year overall survival of $<20 \%(1,2)$.

Prognostic biomarkers are gaining attention as they are able to predict survival and response to treatment of patients with EOC at time of diagnosis, and many gynecologic oncologists have emphasized the need for these markers to select patients for individually-based treatment $(3,4)$. Cancer antigen (CA) 125 is the best known biomarker for ovarian cancer and the only biomarker that is used in daily practice worldwide. It was discovered by Bast et al in 1981 and has been used ever since (5). Clinically, post-treatment serum CA 125 has proven its value and is used for the follow-up of EOC patients $(4,6,7)$. However, the role of preoperative serum CA 125 as a prognostic marker for survival remains questionable. The majority of studies could not demonstrate a prognostic significance (8-12), while other studies found that preoperative serum CA 125 correlated significantly with survival in univariate analysis $(3,13-16)$, although the prognostic effect was lost in multivariate analysis in most of these studies $(3,13,15)$.

EOC typically presents as a large cystic mass which contains variable amounts of fluid (17). Ovarian cyst fluid (oCF) is encapsulated in the ovarian tumor and therefore, it represents a micro-environment which contains compounds directly released by the tumor tissue. Since preoperative punction of oCF might cause tumor spill, it can only be obtained during or after surgery. Therefore, the clinical value of biomarkers in oCF is limited to prediction of survival and/or response to treatment of patients with EOC. A number of studies have compared oCF CA 125 levels between patients with benign and malignant ovarian tumors (18-21). However, to our knowledge, no studies have been published investigating the prognostic value of oCF CA 125 for patients with EOC.

We performed the first study that explored the prognostic value of oCF CA 125 for the prediction of survival of patients with EOC. We also investigated the relation between oCF 
CA 125 levels and important clinicopathological parameters of patients with EOC.

\section{Material and methods}

Patients. In the period between January 2000 and January 2008, ovarian cyst fluid (oCF) was collected from patients who underwent primary surgery for an ovarian tumor at the Radboud University Nijmegen Medical Centre (RUNMC) and stored at our cyst fluid biobank. During or after primary debulking or diagnostic surgery, oCF was collected when a cystic ovarian tumor was diagnosed as malignant by frozen section examination. Cyst fluid collection was never performed during interval debulking surgery. oCF was obtained from 68 patients. Four patients were excluded because final paraffin examination revealed that the origin of the primary tumor was not ovarian derived. Another 10 patients were excluded because the ovarian malignancy was non-epithelial. Included in this study were patients with histologically-proven primary epithelial ovarian cancer (EOC) $(n=54$ patients). Informed consent was obtained from all participants.

Sample collection. Cyst fluid samples of the ovarian tumors were collected by aseptic fine-needle aspiration at the Department of Pathology, immediately after surgical removal of the tumor. Preoperative serum samples of the patients $(n=51)$ were taken within one week prior to surgery. From 3 patients, preoperative serum could not be obtained. After cooled transport to our laboratory, the oCF and blood samples were centrifuged at $3000 \mathrm{x} \mathrm{g}$ for $10 \mathrm{~min}$ and the supernatant was aliquoted and stored at $-35^{\circ} \mathrm{C}$ until use.

CA 125 measurements. CA 125 measurements in oCF and serum were performed with the commercially available CA 125 assay (AxSYM, Abbott Laboratories, Chicago, IL, USA) with a minimum detectable concentration of $2 \mathrm{U} / \mathrm{ml}$. This assay is based on microparticle enzyme immunoassay technology and uses the murine monoclonal antibody OC 125 as catching antibody. Determination of CA 125 was carried out without prior knowledge of the patients' clinical outcome.

Clinicopathological characteristics. Complete pathological reports of all EOC patients were reviewed for correct histopathological diagnosis (primary ovarian carcinoma, histological tumor subtype and tumor grade) by one pathologist (J.B.), specialized in gynaecological oncology. Surgery and FIGO staging was always performed by a gynaecologist specialized in oncology from the RUMCN. From the medical records, the following clinicopathological characteristics were retrospectively retrieved: age at diagnosis, FIGO stage, residual tumor after surgery, tumor recurrence and date of death. For some patients, information about one or more clinicopathological parameters could not be retrieved (Table I). Staging was performed according to the criteria of the International Federation of Gynaecologists and Obstetricians (FIGO) (22). Patients were divided into two groups: patients with early stage (FIGO stage Ia-IIa) and patients with advanced stage (FIGO IIb-IV) EOC (23).

Histopathological tumor type and grade were classified according to the World Health Organization (WHO) criteria
(24). Existence of residual tumor after surgery was divided into two groups by the criteria for optimal cytoreduction (25); patients who had no residual disease or a residual lesion $<1 \mathrm{~cm}$, and patients with a suboptimal debulking of one or more centimeters residual tumor. Recurrence of disease was defined as a measurable lesion during follow-up evaluated by computed tomography, magnetic resonance imaging and/or ultrasonography.

Statistical analysis. Statistical analysis was carried out using SPSS 16.0.2 software (SPSS Benelux BV, Gorinchem, The Netherlands). Correlations between oCF CA 125 and serum CA 125 values were analyzed by Spearman's rank correlation testing. Normality of value distributions was assessed using Kolmogorov-Smirnov testing, and obtained by normal-log (Ln) transformation of both serum and oCF CA125 levels. Differences in levels of CA125 in serum or oCF samples from EOC patients categorized by clinicopathological characteristics, as grouping variables, were assessed with parametric Student's t-tests or ANOVA with post-hoc Tukeys HSD tests where appropriate. The median of CA125 values (for both serum and oCF CA 125) was used as the cut-off value for dichotomizing oCF and serum CA 125. Diseasefree survival (DFS) time was used as follow-up end-point and was defined as the time interval from the date of surgery to the date of recurrence or death, and censored at last follow-up. Survival curves were generated using the method of Kaplan-Meier. Equality of survival distributions was tested using log-rank testing, univariate and multivariate Cox regression analysis.

\section{Results}

Study population. The patient's age at diagnosis ranged from 32 to 89 years, with a median age of 56 years. Numbers of patients in various clinicopathological subgroups are listed in Table I. Serous carcinomas were found in 24 patients $(44 \%)$, 14 patients had mucinous carcinomas $(26 \%)$, and 8 patients had endometrioid type carcinomas $(15 \%)$. Of the remaining 8 patients in the category 'other', 2 patients had clear cell carcinomas (4\%), 1 patient had an undifferentiated adenocarcinoma $(2 \%)$ and 5 patients had ovarian adenocarcinomas not otherwise specified (NOS) (9\%). Of the 19 patients with early FIGO stage, 11 had Ia, 1 had Ib and 7 patients had FIGO Ic. Of the 34 patients with advanced FIGO stage, 2 had IIb, 2 had IIc, 3 had IIIa, 8 had IIIb, 13 had IIIc, and 6 patients had FIGO stage IV. For 1 patient, FIGO stage could not be obtained. Of the total number of 54 patients, 31 (58\%) were treated with 6 courses of adjuvant platinum-based chemotherapy after primary surgery, $5(9 \%)$ patients received neo-adjuvant chemotherapy before interval surgery, and $18(33 \%)$ patients did not receive chemotherapy at all. Median follow-up time was 42 months (range: 10-120 months). None of the patients was lost to follow-up. DFS ranged from 1 to 120 months, with a median of 16 months. Within the followup period, 25 patients (46\%) showed recurrent disease and 17 patients died (32\%).

CA 125 levels in oCF and preoperative serum. Values for CA125 in oCF $(n=54)$ of patients with EOC ranged from 590 

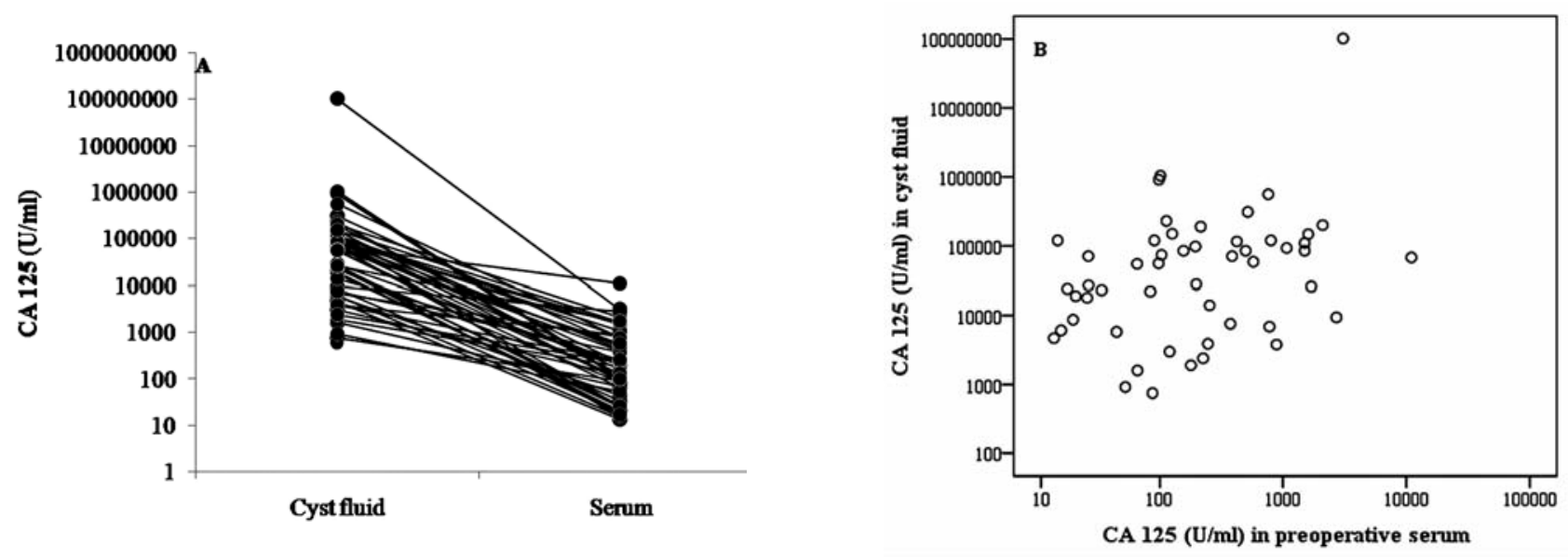

Figure 1. (A) Serum and oCF levels of CA 125 in patients with epithelial ovarian cancer. CA 125 in oCF is always higher than in serum (p<0.001, Student's t-test). (B) CA 125 levels between oCF and serum only moderately correlate ( $\mathrm{p}=0.016, \mathrm{R}=0.337$, Spearman's correlation test).

Table I. Association of CA $125(\mathrm{U} / \mathrm{ml})$ in oCF and serum and clinicopathologically defined patient categories.

\begin{tabular}{|c|c|c|c|c|c|}
\hline Category & $\mathrm{n}(\%)$ & $\begin{array}{c}\text { CA } 125 \text { oCF }(\mathrm{U} / \mathrm{ml}) \\
\text { Median (interquartile range) }\end{array}$ & $\mathrm{P}^{\mathrm{a}}$ & $\begin{array}{c}\text { CA } 125 \text { serum }(\mathrm{U} / \mathrm{ml}) \\
\text { Median (interquartile range) }\end{array}$ & $\mathrm{Pa}^{\mathrm{a}}$ \\
\hline Age & & & 0.905 & & 0.858 \\
\hline$\leq 56$ years & $27(50)$ & $68,000(111,300)$ & & $138(729)$ & \\
\hline$>56$ years & $27(50)$ & $55,000(116,100)$ & & $195(596)$ & \\
\hline Histology & & & $<0.001$ & & 0.014 \\
\hline Serous & $24(44)$ & $79,500(152,250)$ & $\mathrm{a}$ & $387(1,187)$ & $\mathrm{a}$ \\
\hline Mucinous & $14(26)$ & $5,250(10,200)$ & $\mathrm{b}$ & $59(111)$ & $\mathrm{b}$ \\
\hline Endometrioid & $8(15)$ & $109,000(85,000)$ & $\mathrm{a}$ & $387(647)$ & \\
\hline Other & $8(15)$ & $78,000(198,325)$ & $\mathrm{a}$ & $176(1,218)$ & \\
\hline FIGO stage & & & 0.076 & & $<0.001$ \\
\hline Ia-IIa & $19(35)$ & $20,500(87,150)$ & & $66(94)$ & \\
\hline IIb-IV & $34(63)$ & $71,000(137,300)$ & & $387(1,374)$ & \\
\hline Unknown & $1(2)$ & & & & \\
\hline Tumor grade & & & 0.038 & & 0.007 \\
\hline 1 & $14(26)$ & $5,950(21,275)$ & $\mathrm{a}$ & $55(117)$ & $\mathrm{a}$ \\
\hline 2 & $16(30)$ & $21,500(95,675)$ & & $321(738)$ & \\
\hline 3 & $20(37)$ & $89,000(123,250)$ & $\mathrm{b}$ & $225(1,492)$ & $\mathrm{b}$ \\
\hline Other/unknown & $4(7)$ & $322,500(845,500)$ & & $300(650)$ & \\
\hline Residual disease & & & 0.188 & & 0.109 \\
\hline$<1 \mathrm{~cm}$ & $43(80)$ & $24,000(114,200)$ & & 153 (492) & \\
\hline$\geq 1 \mathrm{~cm}$ & $11(20)$ & $85,000(164,000)$ & & $198(1,186)$ & \\
\hline
\end{tabular}

aP-value of Student's t-test or ANOVA of Ln normalized values; a and b denote categories that differ significantly in post-hoc Tukey's HSD analysis.

up to $10,200,000$ (median 55,500) $\mathrm{U} / \mathrm{ml}$. Preoperative serum CA 125 values could be obtained from 51 patients, and ranged from 13 to 11,000 (median 179) $\mathrm{U} / \mathrm{ml}$. CA 125 in oCF was always higher than in serum $(\mathrm{p}<0.001$, Student's t-test, Fig. 1A). Serum and oCF CA 125 within single patients were moderately correlated $(\mathrm{R}=0.337, \mathrm{p}=0.016$, Spearman's correlation test, Fig. 1B).
Differences between histopathological patient categories. Table I lists the association of CA125 in oCF and CA 125 in preoperative serum with different clinicopathological patient characteristics. CA 125 in oCF was significantly lower in patients with mucinous tumors compared to patients with other histological subtypes $(\mathrm{p}<0.001)$, and was significantly lower in patients with well-differentiated tumors compared to 
Table II. Univariate Cox regression analysis of clinicopathological parameters and oCF CA 125 as prognostic factors for DFS in patients with EOC.

\begin{tabular}{lcc}
\hline Variable & HR $(95 \% \text { CI })^{\mathrm{a}}$ & P-value \\
\hline Age & & 0.493 \\
$\leq 56$ years & 1.00 & \\
$>56$ years & $1.30(0.61-2.79)$ & \\
Histology & & $<0.001$ \\
$\quad$ Serous & $10.96(2.28-52.67)$ & 0.003 \\
Mucinous & 1.00 & \\
Endometrioid & $1.30(0.12-14.72)$ & 0.834 \\
Other & $11.17(2.25-55.51)$ & 0.003 \\
FIGO stage & & $<0.001$ \\
Ia-IIa & 1.00 & \\
IIb-IV & $31.12(4.11-235)$ & \\
Tumor grade & 1.00 & 0.025 \\
$\quad 1$ & & \\
$2+3$ & $60.61(1.68-2191)$ & \\
Residual disease & 1.00 & $<0.001$ \\
$<1$ cm & & \\
$\geq 1$ cm & 1.00 & 0.009 \\
CA125 serum & $(2.44-13.93)$ & \\
$<$ median & $3.11(1.29-7.51)$ & \\
$>$ median & 1.00 & 0.005 \\
CA125 oCF & $3.40(1.45-7.97)$ & \\
$<$ median & & \\
$>$ median & & \\
\hline
\end{tabular}

${ }^{\text {a}}$ Hazard ratio (95\% confidence interval); 1.00 , reference category.

patients with poorly-differentiated tumors $(\mathrm{p}=0.038)$. Serum CA 125 was also lower in patients with mucinous tumors $(\mathrm{p}=0.014)$ compared to patients with serous tumors and correlated with tumor grade $(\mathrm{p}=0.007)$ as well. In addition, serum CA 125 was significantly higher in patients with advanced FIGO stage $(\mathrm{p}<0.001)$ compared to patients with early FIGO stage.

Survival analysis. Fig. 2 shows the Kaplan-Meier DFS curves of the patients with oCF CA 125 median values $>55,500 \mathrm{U} / \mathrm{ml}$ and $<55,500 \mathrm{U} / \mathrm{ml}$ (Fig. 2). High levels of oCF CA 125 were significantly associated with a poor DFS $(\mathrm{p}=0.002$, log-rank test). Table II shows the hazard ratio with 95\% CI, using the univariate proportional hazard model. For the variables 'FIGO stage', 'tumor grade' and 'serum CA $125^{\prime}$, one, four and three cases were missing, respectively. Advanced FIGO stage, serous histology, high tumor grade, a suboptimal debulking, high preoperative serum CA 125 and high oCF CA 125 levels were significant predictors of a poor DFS. However, using the multivariate proportional hazard model with selection procedure, FIGO stage was the only independent predictor that had impact on DFS $(\mathrm{HR}=87.1$;

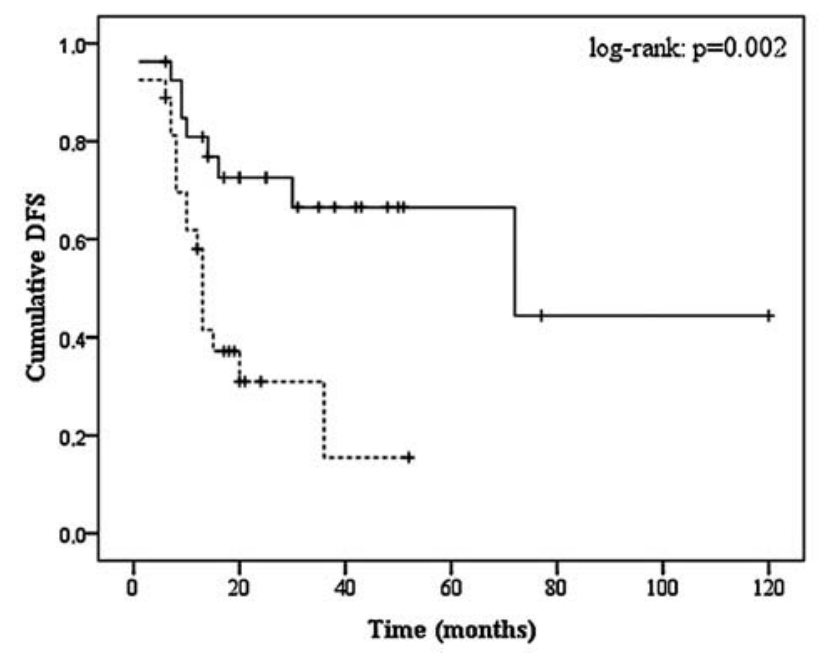

Figure 2. Kaplan-Meier estimates of disease-free survival (DFS) of patients with $\operatorname{EOC}(n=54)$. (A) Low oCF CA 125 values $(n=27 ;<$ median $)$ and high oCF CA 125 values $(n=27 ;>$ median) are represented by the solid and broken line, respectively. Vertical bars indicate patients with censored data.

95\% CI: $1.11-6845 ; \mathrm{p}=0.045)$. As a result, the other factors did not contribute additionally to FIGO stage to predict the time to recurrence.

\section{Discussion}

This is the first study that explored if CA 125 in oCF could be of prognostic value for patients with EOC. Ovarian CF is in close contact with the ovarian tumor epithelium and can be easily obtained by fine-needle aspiration after removal of the ovarian tumor at primary surgery. CA 125 measurement in oCF is possible with the standard CA 125 assay and therefore, seems to be applicable in clinical practice. In the present study high levels of oCF CA 125 were significantly associated with a poor prognosis of EOC patients. In addition, CA 125 in oCF did not show any relationship with FIGO stage and/or debulking status, which have been shown to be the most important prognostic factors for EOC (3). These findings suggested an independent prognostic role for oCF CA 125. However, multivariate survival analysis showed that FIGO stage was the only independent predictor of the time to recurrence. Therefore, we concluded that CA 125 in oCF was not of additional value as a predictive marker for patients with EOC.

Prognostic markers are of major importance for the clinical management of patients with EOC and therefore, identification of the group of patients with a poor disease outcome should occur as early as possible. Currently, serum CA 125 is used during chemotherapeutic treatment to monitor response. It has also proven its value to predict recurrence and disease progression after primary therapy $(4,6,7)$. However, the role of preoperative serum CA 125 as a prognostic tumor marker remains unclear as most studies could not show a significantly independent predictive effect $(3,4,9,11-14,26)$. We found that preoperative serum CA 125 was a significant predictor of DFS in univariate analysis. However, it correlated strongly with FIGO stage, which also has been demonstrated in previous studies $(3,9,14,27)$. Therefore, similar to oCF 
CA 125 , preoperative serum CA 125 in the present study was not of additional prognostic value either.

Only two reports have evaluated the prognostic value of CA 125 expression in ovarian tissue. De la Cuesta et al (28) found that patients with a high expression of the analyte had a significantly poorer prognosis compared to patients with no expression. More recently, Høgdall et al (8) reported on a large series of ovarian tissue samples that increased tissue expression of CA 125, as determined by immunohistochemistry, was related to poor prognosis, but only in the group of patients with advanced stage EOC. In the present study, we also found that patients with high levels of CA 125 in oCF had a significantly poorer prognosis and also that these oCF CA 125 levels were not related to FIGO stage. Therefore, we believe that oCF CA 125 might give a representative reflection of the tumor tissue biology.

The present study demonstrated that oCF levels varied between different histopathological subtypes. More specifically, mucinous tumors contained significantly lower amounts of oCF CA 125 than all other subtypes. It has been shown previously that the proportion of CA 125-producing cells differs between EOCs due to its well-known variety of histopathological subtypes $(8,20,29)$, of which mucinous tumors produce less CA 125 (8). In their series of 584 ovarian cancer tissue samples, Høgdall et al found CA 125 expression in 12, 40,65 and $85 \%$ of mucinous, clear cell, endometrioid and serous samples, respectively (8).

The mechanism of CA 125 release from epithelial cells of the ovary into oCF or serum has been poorly understood. Jacobs et al (30) reported that elevation of preoperative serum CA 125 is not always related to the production of the CA 125 analyte in ovarian tissue. In FIGO stage I EOC, when the tumor is still confined to the ovaries, the phenomenon of high tissue CA 125 levels in association with normal serum CA 125 levels has been demonstrated (30). A similar observation was found in patients with benign ovarian tumors, where high CA 125 levels in oCF were accompanied by low serum CA 125 levels (20). This might suggest that CA 125 is released into the serum only in case of extensive infiltrative growth and wide-spread malignancy, probably because of loss of permeability of the basement membrane and the substantial contribution of metastatic lesions to the CA 125 production (20). Therefore, preoperative serum $\mathrm{CA} 125$ seems to be a marker for disease progression, which might explain its high correlation with FIGO stage of disease. On the other hand, CA 125 in oCF seems to result from a more continuous production brought about by the malignant ovarian epithelium. In the present study, oCF CA 125 was only correlated with histology and grade, which are pathological characteristics of the tumor tissue itself.

This study was performed to explore the predictive possibilities of analysis of CA 125 in cyst fluid of patients with EOC. Our retrospective study comprised a relatively small and heterogeneous cohort of patients with EOC. Although we found that high levels of CA 125 were significantly associated with a poor DFS in univariate analysis, a prospective study with more uniform samples and increased power is required to replicate our findings. Based on the results of the present explorative study, we suppose that analyzing CA 125 in oCF has no additional value in prediction of recurrence for patients with EOC.

\section{References}

1. Jemal A, Siegel R, Ward E, et al: Cancer statistics, 2008. CA Cancer J Clin 58: 71-96, 2008.

2. Colombo N, Van GT, Parma G, et al: Ovarian cancer. Crit Rev Oncol Hematol 60: 159-179, 2006.

3. Tingulstad S, Skjeldestad FE, Halvorsen TB and Hagen B: Survival and prognostic factors in patients with ovarian cancer. Obstet Gynecol 101: 885-891, 2003.

4. Hogdall E: Cancer antigen 125 and prognosis. Curr Opin Obstet Gynecol 20: 4-8, 2008.

5. Bast RC, Jr, Feeney M, Lazarus H, Nadler LM, Colvin RB and Knapp RC: Reactivity of a monoclonal antibody with human ovarian carcinoma. J Clin Invest 68: 1331-1337, 1981.

6. Yedema CA, Kenemans P, Voorhorst F, et al: CA 125 half-life in ovarian cancer: a multivariate survival analysis. Br J Cancer 67: 1361-1367, 1993

7. Duffy MJ, Bonfrer JM, Kulpa J, et al: CA125 in ovarian cancer: European Group on Tumor Markers guidelines for clinical use. Int J Gynecol Cancer 15: 679-691, 2005.

8. Hogdall EV, Christensen L, Kjaer SK, et al: CA125 expression pattern, prognosis and correlation with serum CA125 in ovarian tumor patients. From The Danish 'MALOVA' Ovarian Cancer Study. Gynecol Oncol 104: 508-515, 2007.

9. Rossi AC, Di VG, Cormio G, et al: A retrospective study of preoperative CA 125 levels in 82 patients with ovarian cancer. Arch Gynecol Obstet 269: 263-265, 2004.

10. Gadducci A, Zola P, Landoni F, et al: Serum half-life of CA 125 during early chemotherapy as an independent prognostic variable for patients with advanced epithelial ovarian cancer: results of a multicentric Italian study. Gynecol Oncol 58: 42-47, 1995.

11. Cruickshank DJ, Fullerton WT and Klopper A: The clinical significance of pre-operative serum CA 125 in ovarian cancer. Br J Obstet Gynaecol 94: 692-695, 1987.

12. Sevelda P, Schemper M and Spona J: CA 125 as an independent prognostic factor for survival in patients with epithelial ovarian cancer. Am J Obstet Gynecol 161: 1213-1216, 1989.

13. Geisler JP, Miller GA, Lee TH, Harwood RM, Wiemann MC and Geisler HE: Relationship of preoperative serum CA-125 to survival in epithelial ovarian carcinoma. J Reprod Med 41: 140-142, 1996.

14. Makar AP, Kristensen GB, Kaern J, Bormer OP, Abeler VM and Trope CG: Prognostic value of pre- and postoperative serum CA 125 levels in ovarian cancer: new aspects and multivariate analysis. Obstet Gynecol 79: 1002-1010, 1992.

15. Nagele F, Petru E, Medl M, Kainz C, Graf AH and Sevelda P: Preoperative CA 125: an independent prognostic factor in patients with stage I epithelial ovarian cancer. Obstet Gynecol 86: 259-264, 1995

16. Cooper BC, Sood AK, Davis CS, et al: Preoperative CA 125 levels: an independent prognostic factor for epithelial ovarian cancer. Obstet Gynecol 100: 59-64, 2002.

17. Kurman RJ: Blaustein's pathology of the female genital tract. 5th edition. Springer-Verlag, New York, 2002.

18. Menczer J, Ben-Baruch G, Moran O and Lipitz S: Cyst fluid CA 125 levels in ovarian epithelial neoplasms. Obstet Gynecol 81: 25-28, 1993.

19. Sedlaczek P, Frydecka I, Gabrys M, Van DA, Einarsson R and Harlozinska A: Comparative analysis of CA125, tissue polypeptide specific antigen, and soluble interleukin-2 receptor alpha levels in sera, cyst, and ascitic fluids from patients with ovarian carcinoma. Cancer 95: 1886-1893, 2002.

20. Fleuren GJ, Nap M, Aalders JG, Trimbos JB and de Bruijn HW: Explanation of the limited correlation between tumor CA 125 content and serum CA 125 antigen levels in patients with ovarian tumors. Cancer 60: 2437-2442, 1987.

21. Candido Dos Reis FJ, Moreira de AJ and Bighetti S: CA 125 and vascular endothelial growth factor in the differential diagnosis of epithelial ovarian tumors. Gynecol Obstet Invest 54: 132-136, 2002.

22. Pecorelli S, Benedet JL, Creasman WT and Shepherd JH: FIGO staging of gynecologic cancer. 1994-1997 FIGO Committee on Gynecologic Oncology. International Federation of Gynecology and Obstetrics. Int J Gynaecol Obstet 65: 243-249, 1999. 
23. Vergote I and Trimbos BJ: Treatment of patients with early epithelial ovarian cancer. Curr Opin Oncol 15: 452-455, 2003.

24. Servov SF, Scully RE and Sobin LH: International histologic classification of tumors. No. 9: Histologic typing of ovarian tumors. World Health Organization, Geneva, 1973.

25. Chi DS, Eisenhauer EL, Lang J, et al: What is the optimal goal of primary cytoreductive surgery for bulky stage IIIC epithelial ovarian carcinoma (EOC)? Gynecol Oncol 103: 559-564, 2006.

26. Gadducci A, Cosio S, Tana R and Genazzani AR: Serum and tissue biomarkers as predictive and prognostic variables in epithelial ovarian cancer. Crit Rev Oncol Hematol 69: 12-27, 2009

27. Clark TG, Stewart ME, Altman DG, Gabra H and Smyth JF: A prognostic model for ovarian cancer. Br J Cancer 85: 944-952, 2001 .
28. de la CR, Maestro ML, Solana J, et al: Tissue quantification of CA 125 in epithelial ovarian cancer. Int J Biol Markers 14: 106-114, 1999.

29. Kabawat SE, Bast RC, Welch WR, Knapp RC and Colvin RB: Immunopathologic characterization of a monoclonal antibody that recognizes common surface antigens of human ovarian tumors of serous, endometrioid, and clear cell types. Am J Clin Pathol 79: 98-104, 1983.

30. Jacobs I and Bast RC Jr: The CA 125 tumour-associated antigen: a review of the literature. Hum Reprod 4: 1-12, 1989. 